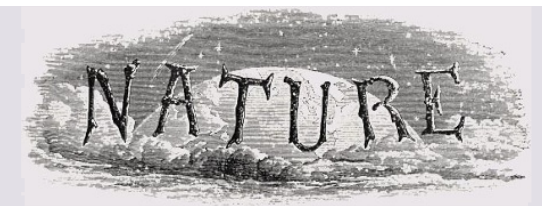

100 YEARS AGO

Nothing is worse than fog at sea. A storm may cause discomfort, an accident may cause delay, but in neither case does the traveller feel so helpless as when his vessel is completely shut in by a dense fog. To lessen the danger which then exists, Prof. E. C. Pickering, the

Director of the Harvard College

Observatory, suggests, in a pamphlet just received, a method of determining the position of a vessel in a fog, based upon the velocity of sound. If two fog-horns of different pitch be placed at equal distances from the middle of a channel or entrance to a harbour, and be sounded simultaneously at regular intervals of about a minute, it will be evident that a captain of a vessel will be able to locate his position with fair accuracy by noting when the sounds of the horns are heard. If the two sounds are heard at the same instant the vessel will be in the middle of the channel, and if they are heard after one another it would be possible to judge from the interval between the two how much the vessel is out of the middle of the channel.

From Nature 10 June 1897.

\section{YEARS AGO}

On June 2, 1897, in his well-known address to the Victoria Institute on "The Age of the Earth", Lord Kelvin said: "I must first ask you to excuse my giving you all my depths, heights, and distances, in terms of the kilometre, being about six-tenths of that very inconvenient measure the English statute mile, which with all the other monstrosities of our British metrical system, will, let us hope, not long survive the legislation of our present

Parliamentary session destined to honour the sixty years' Jubilee of Queen

Victoria's reign by legalising the French metrical system for the United Kingdom". The Weights and Measures (Metric System) Act was passed, and it is no longer a punishable offence for a tradesman to have in his possession a weight or measure of the decimal system. But with all the "monstrosities of our British metrical system" still surviving, it is interesting to recall the fiftieth anniversary of Lord Kelvin's unfulfilled hope. - 'Lord Kelvin and the British metrical system.'

From Nature 14 June 1947.

\title{
The Solar System
}

\section{The frontier beyond Neptune}

Glen R. Stewart

U ntil five years ago, Pluto was viewed as a lonely outpost at the edge of the Solar System. Then in September 1992, Dave Jewitt and Jane Luu ${ }^{1}$ discovered the first of a population of small objects beyond Neptune. These objects, which make up what has come to be known as the Kuiper belt, are in nearly-circular orbits that have remained largely unchanged since the formation of the outer planets ${ }^{2}$. In last week's Nature ${ }^{3}$, Luu et al. presented the discovery of a new type of trans-neptunian object, with a very large semimajor axis, and is probably one of the first of a population of objects that were scattered into this region by Neptune. Its discovery marks an important stage in our understanding of planet formation and the structure of the outer Solar System, and apparently confirms the existence of a 'scattered disk' - a new component of the Solar System predicted by Duncan and Levison in tomorrow's Science ${ }^{4}$.

Although speculation on the existence of the Kuiper belt dates back almost 50 years ${ }^{5,6}$, serious work on the topic did not start until Fernández ${ }^{7}$ invoked it as a possible source of the short-period comets (those with orbital periods of less than 200 years). Dynamical simulations ${ }^{8}$ showed that a comet belt beyond Neptune is the most plausible source for the low-inclination, Jupiter-family comets. The first Kuiper-belt object, an icy body about $250 \mathrm{~km}$ across, was discovered in 1992.

So far, 40 Kuiper-belt objects have been $1_{1996 \mathrm{TL}_{66}}$. It has an unusually eccentric orbit

discovered with ground-based telescopes. As only a small fraction of the sky has been searched, these 40 imply $^{9}$ a total population of 100 -km objects of about 70,000, assuming that most objects are near the ecliptic (the plane of the Earth's orbit). Of the 40 known objects, 21 have well-determined orbits, and fall into two main classes. Inside $41 \mathrm{AU}$ (Neptune is at $30 \mathrm{AU}$ ), they all have eccentricities between 0.1 and 0.3 , and they are in meanmotion resonances with Neptune - that is, the ratio of their orbital period to Neptune's is a ratio of small integers. Beyond $\sim 41 \mathrm{AU}$, they do not tend to be in mean-motion resonances and mostly have eccentricities less than 0.1. All the well-observed Kuiper-belt objects have semimajor axes of less than 50 $\mathrm{AU}$ and inclinations less than $20^{\circ}$.

To explain this surprisingly intricate structure, models have included the effects of the migration of Uranus and Neptune as they formed ${ }^{10}$, the effects of a massive protoplanetary disk ${ }^{11}$, and the effects of individual massive planetesimals ${ }^{12}$ on the orbits of Kuiper-belt objects. These models predict that Kuiper-belt objects will have eccentricities in the range $0-0.3$ and inclinations of $0-15^{\circ}$.

The new prediction of a 'scattered disk' comes from simulations ${ }^{4}$ of the behaviour of objects that originated in the region between Uranus and Neptune or that were perturbed by Neptune from the inner edge of the Kuiper belt. Some of these objects can come close to Neptune and get scattered into eccentric orbits outside its orbit. The long-
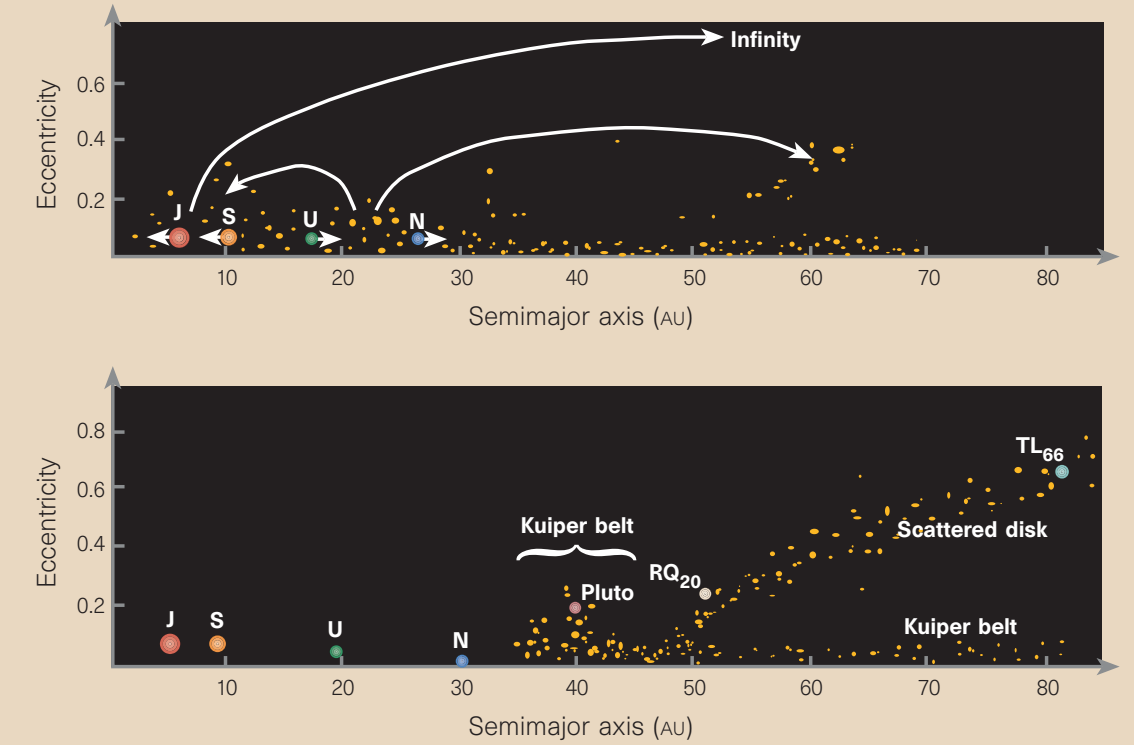

Figure 1 Creation of the scattered disk. Jupiter and Saturn can scatter nearby comets out of the Solar System, but Uranus and Neptune are gentler, and send some comets into long-lived orbits with high eccentricity and semimajor axis. The newly discovered $1996 \mathrm{TL}_{66}$ may be a member of this scattered disk. (Adapted from ref. 4.) 
range gravitational effects of the planets can lift the orbits of these objects beyond the outer edge of the planetary system, protecting them from close encounters with the planets. Therefore, many of these objects can survive in these eccentric orbits for the 4.6billion-year age of the Solar System, forming a scattered disk.

Duncan and Levison's numerical simulations ${ }^{4}$ indicate that objects in the scattered disk tend to have more eccentric and/or inclined orbits than those in the Kuiper belt. Two recently discovered trans-neptunian objects may be the first members of the scattered disk to be found. The first, $1996 \mathrm{RQ}_{20}$, was discovered in September 1996 by E. Helin, D. Brown and D. Rabinowitz. Its semimajor axis is $47 \mathrm{AU}$, its eccentricity is 0.3 , its inclination is $32^{\circ}$, and it is about $300 \mathrm{~km}$ across $^{13}$. The newly discovered second object, $1996 \mathrm{TL}_{66}$, is estimated ${ }^{3}$ to have a semimajor axis of $84 \mathrm{AU}$, an eccentricity of 0.58 , an inclination of $24^{\circ}$, and to be about $490 \mathrm{~km}$ across. These objects are clearly outside the range of orbital elements predicted for the Kuiper belt: $1996 \mathrm{RQ}_{20}$ because of its inclination, and $1996 \mathrm{TL}_{66}$ because of its eccentricity. However, they could be members of the scattered disk ${ }^{4}$.

With the recent advances in our observational and theoretical understanding of the trans-neptunian region, it is now possible to construct a tentative picture of how the outer Solar System formed and evolved.

Jupiter and Saturn formed early on, when the gaseous component of the solar nebula was still present. As the gas dissipated, it left behind a large number of small, icy bodies that had condensed from it, outside the orbit of Saturn and extending outward perhaps as far as a few hundred AU. These objects collided and gradually grew into larger 'planetesimals'. As the planetesimals increased in mass, they began to gravitationally perturb one another into more eccentric and more inclined orbits about the Sun. The growth of planets was much more rapid inside about 40 $\mathrm{AU}$, leading to the formation of Uranus and Neptune. But outside $40 \mathrm{AU}$ (that is, in the Kuiper belt), the bodies remained relatively small. Pluto may be just the largest Kuiperbelt object.

The young Uranus and Neptune scattered most of the remaining nearby planetesimals, outwards into the trans-neptunian region and inwards towards Jupiter and Saturn (Fig. 1). Usually, the objects scattered outwards eventually returned to the Uranus-Neptune region. In contrast, Jupiter and Saturn are so massive that they can efficiently eject many of these planetesimals out of the planetary system - so there was a net flux of objects towards Jupiter. This inward transport required an outward migration of the orbits of Uranus and Neptune in order to conserve the angular momentum and energy of the system ${ }^{14}$. As
Neptune moved outwards, it may have trapped planetesimals farther out into mean motion resonances ${ }^{10}$. Then Neptune's migration (and other processes ${ }^{11,12}$ ) would have pumped up the eccentricity of these objects to values as large as 0.3 , leading to much of the present Kuiper-belt structure described above.

As mentioned, the objects scattered outwards by Uranus and Neptune can be longlived. After their first encounter with Neptune, about $1 \%$ of them are stored in an extended scattered disk beyond Neptune for the age of the Solar System ${ }^{4}$. There are 31 Earth-masses of material in Uranus and Neptune, and as planet formation is far from $100 \%$ efficient, it is reasonable to expect that a similar amount of mass was initially scattered outwards by Neptune (the efficiency factor is not known to better than a factor of a couple of orders of magnitude). So, crudely, we may expect as much as a few tenths of Earth-masses of material in the scattered disk. This value is consistent with current estimates based on the dynamical models of the scattered disk by Duncan and Levison.

Much of the above picture is speculative, and much of it is likely to change rapidly in the coming years or months as this field matures and more data become available. One reason for this is that only a very small fraction (about $0.06 \%$ ) of the Kuiper-belt and scattered-disk objects have yet been discovered. But the fact remains that the structure of the trans-neptunian region is much more complex, and therefore interesting, than was dreamed of only a few years ago.

Glen R. Stewart is in the Laboratory for Atmospheric and Space Physics, University of Colorado, Boulder, Colorado 80309, USA.

e-mail:glen@ptah.colorado.edu

1. Jewitt, D. \& Luu, J. Nature 362, 730-732 (1993).

2. Weissman, P. R. \& Levison, H. F. in Pluto (eds Stern, S. A. \& Tholen, D. J. ) (Univ. Arizona Press, Tucson, AZ, in the press).

3. Luu, J. et al. Nature 387, 573-575 (1997).

4. Duncan, M. J. \& Levison, H. F. Science 276, 1670-1672 (1997).

5. Edgeworth, K. E. Mon. Not. R. Astron. Soc. 109, 600-609 (1949).

6. Kuiper, G. in Astrophysics: A Topical Symposium (ed. Hynek, J. A.) 357-424 (McGraw-Hill, New York, 1951). 7. Fernández, J. Mon. Not. R. Astron. Soc. 192, 481-491 (1980).

8. Duncan, M., Quinn, T. \& Tremaine, S. Astrophys. J. 328, L69-L73 (1988)

9. Jewitt, D., Luu, J. \& Chen, J. Astron. J. 112, 1225-1238 (1996). 10. Malhotra, R. Astron. J. 110, 420-429 (1995).

11. Levison, H. F., Stern, S. A. \& Duncan, M. J. Icarus (submitted). 12. Morbidelli, A. \& Valsecchi, G. B. Icarus (in the press). 13. Marsden, B. G. Minor Planet Electronic Circulars B19 (1997). 14. Fernández, J. A. \& Ip, W.-H. Icarus 58, 109-120 (1984).

\section{The tinkerer's evolving tool-box}

\section{E. Richard Moxon and David S. Thaler}

T he neo-darwinian synthesis resulted from the realization that mendelian inheritance changed the darwinian model of biological evolution. Darwin assumed that parents' genes blend into each other in their offspring. This was problematical because a fitter variant's genes would blend upon mating, and become diluted into the gene pool. Mendelism solved this paradox by providing a mechanism for descent, whereby individual alleles remain intact and are not blended away through mating. This example shows that knowledge of the specifics of genetics - that is, the mechanisms by which variants are generated - sometimes has a profound effect on evolutionary theory.

Another example of this is now provided by two papers on pages 700 and 703 of this issue. In a theoretical study, Taddei et al. ${ }^{1}$ predict that in asexual, clonal populations of Escherichia coli, the ability to generate mutator alleles (which can lead to an increase in mutation rate) increases the rate at which more fit individuals arise in the population. This occurs even if the mutator alleles remain at a very low frequency. Sniegowski and colleagues ${ }^{2}$ have found that three of 12 independently propagated clonal populations of $E$. coli, which were serially cultured over 10,000 generations and thereby subjected to alternating periods of growth and stasis, had a mutator phenotype which was due to defects in genes involved in DNA repair.

These papers transcend the assumption that mutations are spread more or less evenly through a population: this assumption was only reasonable when mutation was considered to be a direct result of random insults from outside an organism. Mutations are now known to be due to processing of the internal consequences of such damage, as well as to endogenous processes. The internal processing - a part of DNA metabolism — is carried out by gene products whose alleles have profound effects on the generation of variation. Alleles that predispose other genes to (possibly beneficial) mutations may hitchhike ${ }^{3}$, because the mutator allele and the phenotypically selected alleles of other genes are linked, especially in asexual clonal populations.

Taddei and colleagues' ${ }^{1}$ detailed populational analysis, derived using the best numerical parameters available for $E$. coli, shows that mutators are a key aspect of adapative evolution. The implications of their results are strengthened by a previous theoretical model ${ }^{4}$ showing that the effects of mutator alleles become larger as the evolutionary challenge is made more complex. The effect of incorporating mutators in the population increases exponentially as a function of the number of mutations required for a transition from one 